\title{
Search for astrophysical sources of muon neutrinos with 38 years of data from the BUST detector
}

\section{M. Boliev}

Institute for Nuclear Research of the Russian Academy of Sciences

E-mail: boliev2005eyandex.ru

\section{A. V. Butkevich}

Institute for Nuclear Research of the Russian Academy of Sciences

\section{M. Dzaparova}

Institute for Nuclear Research of the Russian Academy of Sciences and Institute of Astronomy of the Russian Academy of Sciences

\section{M. Kochkarov*}

Institute for Nuclear Research of the Russian Academy of Sciences

\section{R. V. Novoseltseva}

Institute for Nuclear Research of the Russian Academy of Sciences

\section{B. Petkov}

Institute for Nuclear Research of the Russian Academy of Sciences and Institute of Astronomy of the Russian Academy of Sciences

\section{P. S. Striganov}

Institute for Nuclear Research of the Russian Academy of Sciences

\section{V. Volchenko}

Institute for Nuclear Research of the Russian Academy of Sciences

\section{A. F. Yanin}

Institute for Nuclear Research of the Russian Academy of Sciences

Baksan Underground Scintillation Telescope is an underground detector located at the Northern Caucasus (Russia). The BUST can detect an astrophysical flux of neutrinos from Galactic sources as an excess of cosmic ray muon neutrinos arriving from the source direction. The search performed using 38 years (live time $=30.9$ ) of the BUST dataset to look for a statistically significant excess of events arriving within a solid angle. No significant excess of events produced by astrophysical sources is found. Since the number of detected events is compatible with the number of expected background events upper limit on the muon neutrino flux is determined.

35th International Cosmic Ray Conference - ICRC2017

10-20 July, 2017

Bexco, Busan, Korea 


\section{Experimental Data}

The Baksan Underground Scintillation Telescope (BUST) [1] is located in the underground laboratory at the effective depth of 850 m.w.e. The detector itself is a parallelepiped $16.7 \times 16.7 \times$ $11 \mathrm{~m}^{3}$, all sides of which are entirely covered by liquid scintillator counters of the standard type $\left(0.7 \times 0.7 \mathrm{~m} \times 0.3 \mathrm{~m}^{3}\right)$. There are also two additional horizontal layers inside, at distances of 3.6 and 7.2 meters from the bottom. Thus the detector consists of four horizontal and four vertical planes, each separated from the other by $\simeq 160 \mathrm{~g} / \mathrm{cm}^{2}$ of absorber. The total number of the detectors is 3180 . Every counter is viewed with one PMT (the $15 \mathrm{~cm}$ diameter photocathode). The construction of BUST allows one to identify tracks of muons crossing the telescope. Separation of arrival directions between up and down hemispheres is made by time-of-flight (TOF) method with time resolution $5 \mathrm{~ns}$ [2]. The angular resolution of the BUST for reconstructed events is about $1.6^{\circ}$.The detection of upward-going muons is performed by means of the time-of-flight method. In first period $(1978$ - 2000) two hardware triggers are used in order to reject downward-going atmospheric muons. Trigger I covers the zenith angle range $95^{\circ}-180^{\circ}$, while trigger II selects horizontal muons in the range $80^{\circ}-100^{\circ}$, for more details see ref $[3,4]$. Since 2000 year no use hardware triggers for select neutrino events. The data used for this analysis have been collected from December of 1978 until June of 2017, for a total of 30.9 live-years. It was found that 1635 events survived these cuts.

\section{Search for astrophysical sources}

In this work we calculate real live-time for selected astrophysical objects. Figure 1 shows dependence of BUST detector live-time for declination $(\delta)$. The search for a neutrino induced signal has been performed within angular windows of $5^{\circ}$ around a exact coordinates of selected astrophysical objects. The size of the window was derived by Monte Carlo simulations of pointlike neutrino sources with power law spectra $(\gamma=2.5)$, and is the cone opening angle including $90 \%$ of the effect. The scattering angle between incident neutrino and daughter muon, multiple Coulomb scattering of the muon in the rock, and the angular resolution of the detector have been taken into account. Table 1 shows the number of upward-going muons observed

\footnotetext{
*Speaker.
} 


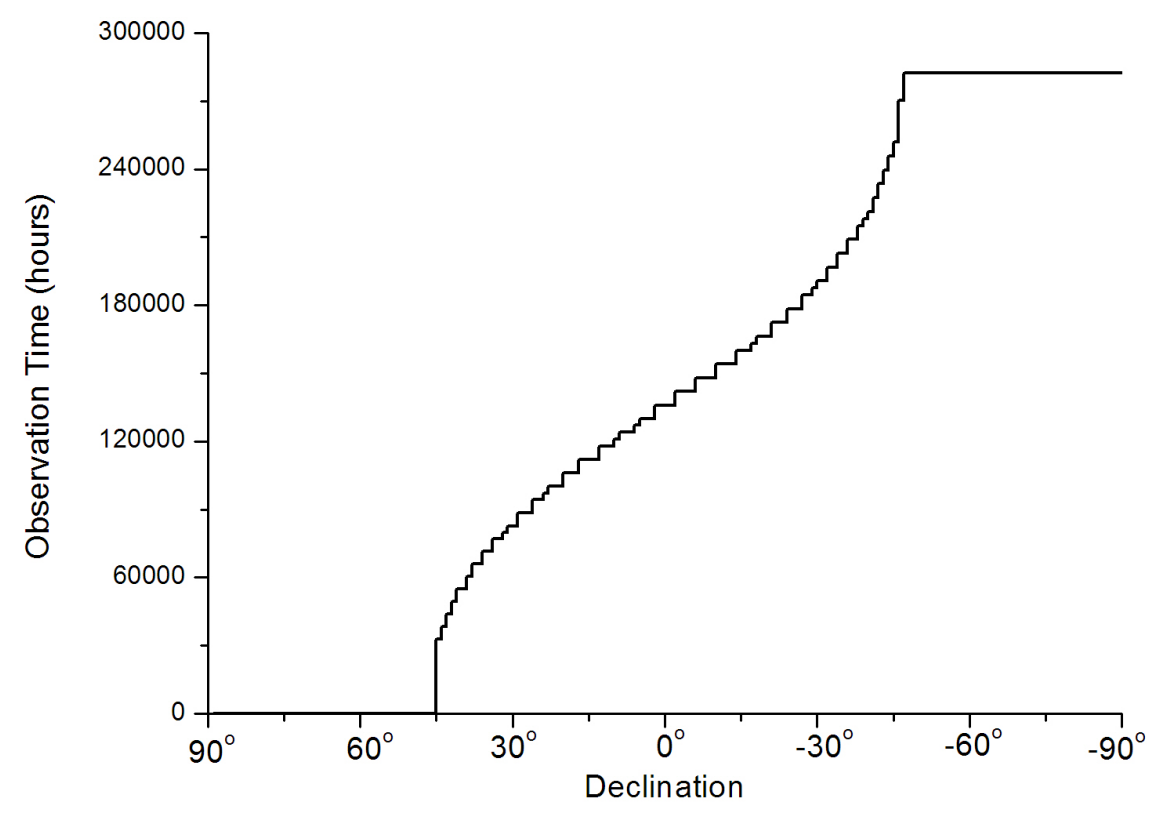

Figure 1: Dependence live-time according by declination - $\delta$

Table 1: Baksan flux limits on astrophysical muon neutrino sources

\begin{tabular}{|c|c|c|c|c|}
\hline Object & $\alpha\left(^{\circ}\right) \delta\left(^{\circ}\right)$ & Background & Events & $\mu$ Flux $10^{-14}\left(\mathrm{~cm}^{-2} \mathrm{~s}^{-1}\right)$ 90\% C.L. \\
\hline G.C. & $265.6-28.9$ & 6.5 & 5 & 0.32 \\
\hline NGC 1952 & $83.6+22$. & 1.9 & 0 & 0.87 \\
\hline Vela X-1 & $135.5-40.3$ & 6.5 & 4 & 0.15 \\
\hline SS433 & $288.0+5.0$ & 2.8 & 1 & 0.63 \\
\hline 3c273 & $187.3+2.0$ & 2.9 & 4 & 0.52 \\
\hline NGC 5128 & $201.4-43.0$ & 6.8 & 5 & 0.15 \\
\hline Cen. X-3 & $170.3-60.6$ & 6.3 & 3 & 0.11 \\
\hline Cyg. X-3 & $307.7+40.8$ & 0.1 & 0 & 0.99 \\
\hline Scorp. X-1 & $245.0-15.6$ & 4.2 & 3 & 0.37 \\
\hline
\end{tabular}

within the window and the expected background, which was evaluated from simulations of the angular distribution of atmospheric neutrino induced muons and randomization of the event arrival times. The neutrino flux of [5] has been used. No evidence is found for any excess, and 90\% C.L. limits for muon fluxes are given in fifth column of Table 1. For this search we have used the entire sample of 1635 events.

\section{References}

[1] Alekseev E. N. et al. 1979 Proc. of the 16th ICRC 10276 
[2] Andreyev Yu. M. et al. 1979 Proc. of the 16th ICRC 10184

[3] Boliev M. M. et al. 1991 Proc. of the 3rd Int. Workshop on Neutrino Telescopes, 235

[4] Boliev M. M. et al. 1995 Proc. of the 24th ICRC, Roma, 1686

[5] Volkova L. V. 1980 Sov. J. Nucl. Phys., 31784 